\title{
TP-AGB stars in population synthesis models
}

\author{
Paola Marigo ${ }^{1}$, \\ Léo Girardi ${ }^{2}$, Alessandro Bressan ${ }^{2}$, Bernhard Aringer ${ }^{2,3}$, \\ Marco Gullieuszik ${ }^{2}$, Enrico V. Held ${ }^{2}$, Martin A.T. Groenewegen ${ }^{4}$, \\ Laura Silva ${ }^{5} \&$ Gian Luigi Granato ${ }^{5}$ \\ ${ }^{1}$ Dept. of Astronomy, University of Padova, Vicolo dell'Osservatorio 3, I-35122 Padova, Italy, \\ email: paola.marigo@unipd.it \\ ${ }^{2}$ Astronomical Observatory of Padova, INAF, Vicolo dell'Osservatorio 5, I-35122 Padova, Italy \\ ${ }^{3}$ Dept. of Astronomy, University of Vienna, Tuerkenschanzstr. 17, A1180 Vienna, Austria \\ ${ }^{4}$ Royal Observatory of Belgium,Ringlaan 3, B-1180 Brussels, Belgium \\ ${ }^{5}$ Astronomical Observatory of Trieste, INAF, Via Tiepolo 11, I-34131 Trieste, Italy
}

\begin{abstract}
In spite of its relevance, the Thermally Pulsing Asymptotic Giant Branch (TPAGB) phase is one of the most uncertain phases of stellar evolution, and a major source of disagreement between the results of different population synthesis models of galaxies. I will briefly review the existing literature on the subject, and recall the basic prescriptions that have been used to fix the contribution of TP-AGB stars to the integrated light of stellar populations. The simplicity of these prescriptions greatly contrasts with the richness of details provided by present-day databases of AGB stars in the Magellanic Clouds, which are now being extended to other nearby galaxies. I will present the first results of an ongoing study aimed at simulating photometry, chemistry, pulsation, mass loss, dust properties of AGB star populations in resolved and un-resolved galaxies. We test our predictions against observations from various surveys of the Magellanic Clouds (DENIS, 2MASS, OGLE, MACHO, Spitzer, and AKARI). I will discuss the implications and outline the plan of future developments.
\end{abstract}

\section{Introduction}

It has been long known that Thermally Pulsing Asymptotic Giant Branch (TP-AGB) stars play a crucial role in many aspects of galaxy evolution, e.g. by providing a significant fraction of the bolometric, near and mid IR light emitted by young to intermediate-age stellar populations (ages $>10^{8} \mathrm{yr}$; Frogel et al. 1990), and efficiently contributing to the enrichment of the interstellar medium in the form of chemical yields (gas and dust) and dark remnants. The products of their rich nucleosynthesis leave easily identifiable signatures in both resolved stellar spectra and integrated galaxy spectra (mainly depending on the photospheric $\mathrm{C} / \mathrm{O}$ ratio).

When applied to nearby systems (e.g. the Galaxy and the Magellanic Clouds) evolutionary population synthesis (EPS) models including TP-AGB stars are useful tools to derive quantitative indications - e.g. mass and metallicity dependence - of fundamental stellar processes such as convection, mass loss, nucleosynthesis, pulsation. On the other hand, in the recent years it has become clear that the study of AGB star populations is relevant over a much wider astrophysical context (see Bruzual, this volume), such as to unveil the SFR history in resolved stellar populations; to break the age-degeneracy that affects the studies of early-type galaxies; to interpret the spectral energy distributions of high-z galaxies in terms of their fundamental parameters (masses and ages). 


\section{TP-AGB Stars in EPS models: techniques}

There are basically three methods to add the AGB phase in EPS models, which are based on the use of $(A)$ stellar isochrones (e.g. Bressan et al. 1998; Bruzual \& Charlot 2003; Marigo et al. 2003); (B) stellar tracks (Groenewegen \& de Jong 1993; Marigo et al. 1999; Mouhcine \& Lançon 2002, 2003); and $(C)$ the fuel consumption theorem by Renzini \& Buzzoni (1986; Maraston 1998, 2005).

Methods $A$ and $B$ both need practically the same ingredients, namely sets of AGB stellar models predicting the evolution of basic stellar parameters (i.e. total mass, core mass, luminosity, effective temperature $T_{\text {eff }}$, surface chemical composition) over wide ranges of initial stellar masses and metallicities. Owing to the heavy requirement of computing time set by the full modelling of the AGB phase, the most convenient way to build up an extended library of AGB stellar tracks is to adopt a synthetic approach, which gives a simplified but flexible and description of the stellar evolution on the AGB. Another important aspect is that synthetic AGB models can be readily calibrated on the basis of basic observables (e.g. carbon star luminosity functions, star counts), hence allowing for a more realistic description of AGB properties. Available stellar isochrones including the whole AGB phase are listed in Table 1, which also outlines a few relevant characteristics, namely: the inclusion or not of the third dredge-up and hot-bottom burning, the way $T_{\text {eff }}$ is obtained depending on the adopted molecular opacities, and the consideration or not of the effect of circumstellar dust on the emitted spectrum.

The fundamental ingredient of method $C$ is the fuel matrix, $F_{\mathrm{AGB}}\left(M_{\mathrm{TO}}, Z\right)$, providing the nuclear fuel burnt during the AGB phase as a function of the turn-off mass $M_{\mathrm{TO}}$ (or equivalently of the age), and the metallicity $Z$ of the corresponding single stellar population.

Then, for all methods the passage from theoretical quantities to observed ones requires the adoption of suitable spectral libraries as a function of $T_{\text {eff }}$, surface gravity and $\mathrm{C} / \mathrm{O}$ ratio, or alternatively tables of bolometric corrections and $T_{\text {eff }}$-color transformations. Finally, one has to specify global properties of the simulated galaxy, such as the initial mass function (IMF), the star formation rate history (SFR), and the age-metallicity relation (AMR).

Concerning the characteristics of the EPS techniques, it should be remarked that both methods $A$ and $B$ are tied, by construction, to the adopted stellar models. On the one hand this brings along all the weak points that affect the theory of stellar evolution, but on the other hand it provides a useful feedback to input prescriptions any time a comparison between predictions and observations is performed. Method $C$ usually adopts an empirical calibration of the AGB nuclear fuel based on observed data for Magellanic Cloud clusters. On the one hand this guarantees accounting for the correct integrated light contribution from AGB stars at LMC and SMC metallicities, but on the other hand it makes it difficult to derive explicit indications on uncertain aspects of the underlying AGB evolution. Moreover, while for methods $A$ and $B$ the resolution element is the single star, so that they can address the study of both integrated and resolved stellar populations, for method $C$ the maximum resolution is given by the simple stellar population (SSP), which limits its application to the integrated properties of unresolved AGB stars.

\section{Synthetic TP-AGB Evolution: ingredients and calibration}

Synthetic AGB models are usually constructed on the basis of some analytical recipe derived from complete modelling of the AGB phase and/or observations - providing, for 
Table 1. Available stellar isochrones including the AGB phase

\begin{tabular}{|c|c|c|c|c|}
\hline Reference & $\mathrm{HBB}^{(\mathrm{a})}$ & $\kappa_{\mathrm{mol}}(\mathrm{b})$ & dust $^{(c)}$ & available at \\
\hline Bertelli et al. 1994, A\&AS 106, 275 & no & scaled-solar & no & http://pleiadi.pd.astro.it \\
\hline Bressan et al. 1998, A\&A 332, 135 & no & scaled-solar & yes & upon request \\
\hline Girardi et al. 2000, A\&AS 141, 371 & no & scaled-solar & no & http://stev.oapd.inaf.it/cmd \\
\hline Marigo \& Girardi 2001, A\&A 377, 132 & yes & scaled-solar & no & http://stev.oapd.inaf.it/cmd \\
\hline Mouhcine 2002, A\&A 394,125 & yes & scaled-solar & yes & upon request \\
\hline Piovan et al. 2003, A\&A 408, 559 & no & scaled-solar & yes & upon request \\
\hline Marigo et al. 2003, A\&A 403, 225 & yes & O-/ C-rich & no & upon request \\
\hline Cioni et al. 2006, A\&A 448, 77 & yes & $\mathrm{O}-/ \mathrm{C}$-rich & no & http://stev.oapd.inaf.it/cmd \\
\hline Cordier et al. 2007, AJ 133, 468 & no & scaled-solar & no & http://www.oa-teramo.inaf.it/BASTI \\
\hline Marigo et al. 2008, A\&A 482,883 & yes & O-/ C-rich & yes & http://stev.oapd.inaf.it/cmd \\
\hline Bertelli et al. 2008 , A\&A 484,815 & yes & $\mathrm{O}-/ \mathrm{C}-\mathrm{rich}$ & no & http://stev.oapd.inaf.it/YZvar \\
\hline
\end{tabular}
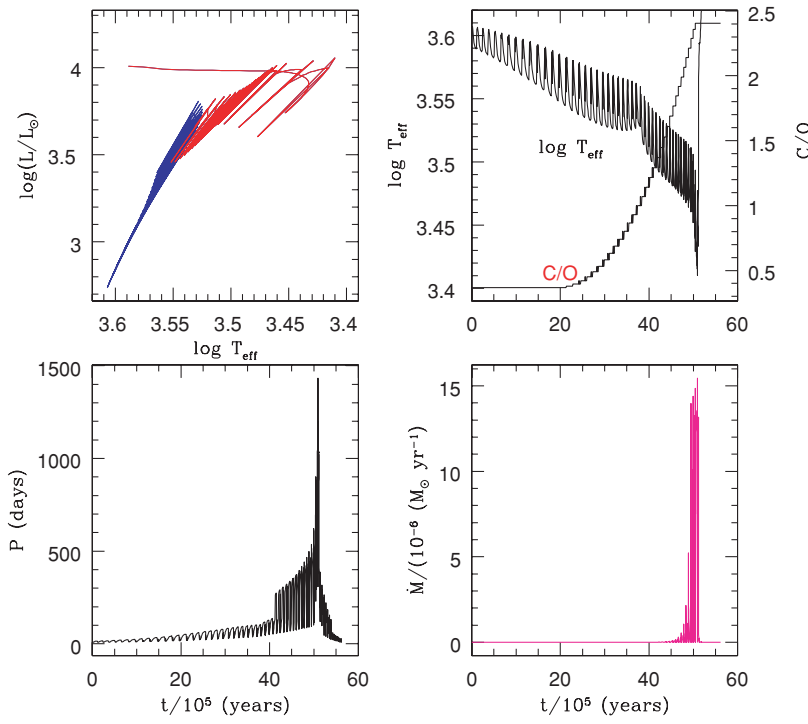

Figure 1. Predicted evolution of luminosity, $T_{\text {eff }}$, surface $\mathrm{C} / \mathrm{O}$ ratio, pulsation period, and mass-loss rate during the TP-AGB evolution of a $1.8 M_{\odot}, Z=0.008$ star, following the prescriptions in Marigo \& Girardi (2007). Low- $T$ opacities are calculated on-the-fly with the ÆSOPUS tool (Marigo \& Aringer 2009), consistently with the changes in the surface chemical composition caused by the third dredge-up. Notice the warming of the track for $0.8 \lesssim \mathrm{C} / \mathrm{O} \lesssim 1$ reflecting a pronounced opacity dip, and the sudden cooling as soon as the star becomes of C-type $(\mathrm{C} / \mathrm{O}>1)$, due to the abrupt change in the main sources of molecular opacity.

instance, the core-mass-luminosity relation, the core-mass -interpulse-period relation, and the mass-loss rates as a function of stellar parameters.

The purely analytical scheme can be significantly complemented with the aid of envelope integrations - from the photosphere down to the surface of the $\mathrm{H}$-exhausted core which allows us e.g. to predict the $T_{\text {eff }}$ along the AGB, as well as to follow the hot-bottom burning (HBB) nucleosynthesis by solving a network of nuclear reactions in the deepest envelope layers of the most massive AGB models (e.g. Marigo et al. 1999).

The TP-AGB evolutionary tracks presented here have been described in Marigo \& Girardi (2007) and Marigo et al. (2008). They include a series of crucial aspects such as: the complex luminosity evolution over the pulse-cycle, the third-dredge-up efficiency as a function of stellar mass and metallicity, the derivation of the $T_{\text {eff }}$ from the integration of stellar envelope models, HBB at the base of convective envelopes, the dramatic changes in low-temperature opacities when the stellar envelopes become C-rich (Marigo 2002), different mass loss descriptions for M- and C-type stars, the dependence of mass loss on 

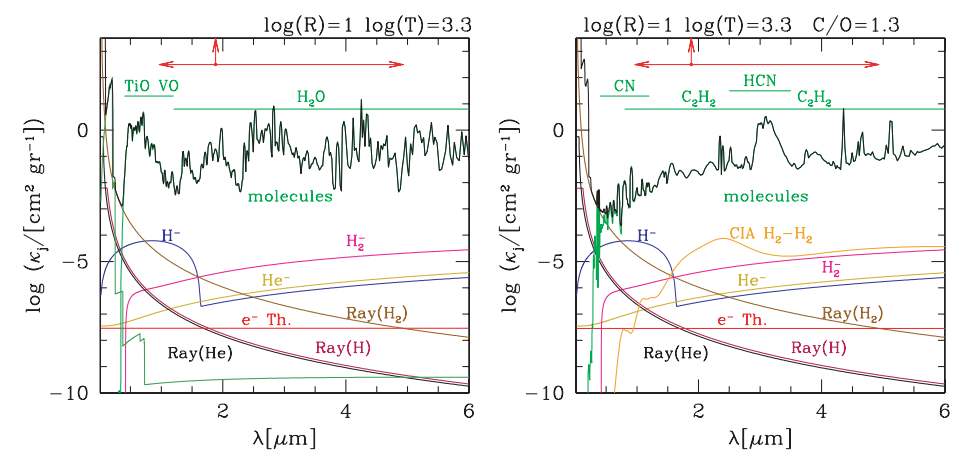

Figure 2. Monochromatic absorption coefficients for several opacity sources as a function of the wavelength, in a gas with $\log T=3.3, \log R=1$, and to values of the $\mathrm{C} / \mathrm{O}$ ratio, corresponding to an O-rich (left panel) and C-rich (right panel) composition. Computations performed with ÆSOPUS (Marigo \& Aringer 2009).

pulsation period, the transition from the first overtone pulsation mode to the fundamental one, etc. One example of is shown in Fig. 1.

The evolutionary tracks contain a few free parameters (mainly related to the third dredge-up) which were calibrated so as that two basic observables of the LMC and SMC were reproduced: the carbon star luminosity functions (Groenewegen 2002), and the Cand M-type lifetimes as a function of turn-off mass as derived from MC star clusters (Girardi \& Marigo 2007a).

A major improvement in the input physics has been recently achieved with the development of the ÆSOPUS (Accurate Equation of State and OPacity Utility Software; Marigo \& Aringer 2009; web interface at http://stev.oapd.inaf.it/aesopus; see example in Fig. 2) for computing the equation of state and the Rosseland mean opacities of the gas at low temperatures $(1500 \leqslant T \leqslant 30000 \mathrm{~K})$ for any chemical composition. The chemistry is presently solved for about 800 species, including almost 500 molecules. ÆSOPUS is being incorporated in the envelope model described in Marigo \& Girardi (2007), allowing correct a evaluation of the atmospheric opacity, hence $T_{\text {eff }}$, as a function of the changes in the surface abundances that characterize the evolution of TP-AGB stars.

\section{From stellar tracks to synthetic samples of AGB stars}

The TP-AGB evolutionary tracks were attached to the Girardi et al. (2000) tracks for the previous evolutionary phases, and used to build the Marigo et al. (2008) isochrones. They consider the reprocessing of radiation by dust in the circumstellar envelopes of masslosing stars. The web interface in http://stev.oapd.inaf.it/cmd provides these isochrones - and their derivatives, such as luminosity functions and integrated magnitudes - for more than 25 optical-to-far-infrared photometric systems, including those more useful to the study of MC AGB stars (e.g. OGLE, DENIS, 2MASS, Spitzer IRAC+MIPS, and AKARI).

In the process of building isochrones, we have used the sequences of synthetic C star spectra from Loidl et al. (2001). These cover well the $T_{\text {eff }}$ range of $\mathrm{C}$ stars in the Magellanic Clouds and in the Milky Way galaxy, but not the wide intervals of surface gravities and $\mathrm{C} / \mathrm{O}$ ratios expected for such stars. Moreover, they have been computed for $\mathrm{C}$ stars of initial solar metallicity. We have recently extended the database of synthetic $\mathrm{C}$ star spectra, using the COMARCS code (Aringer et al. 2009), covering a wide range of metallicities and $\mathrm{C} / \mathrm{O}$ ratios. Spectra and corresponding tables of bolometric corrections are now available at http://stev.oapd.inaf.it/synphot/Cstars. An example of the new synthetic photometry is given in Fig. 3 .

This set of theoretical models have been included in the TRILEGAL code (Girardi et al. 2005) for simulating the photometry of resolved stellar populations. The code 

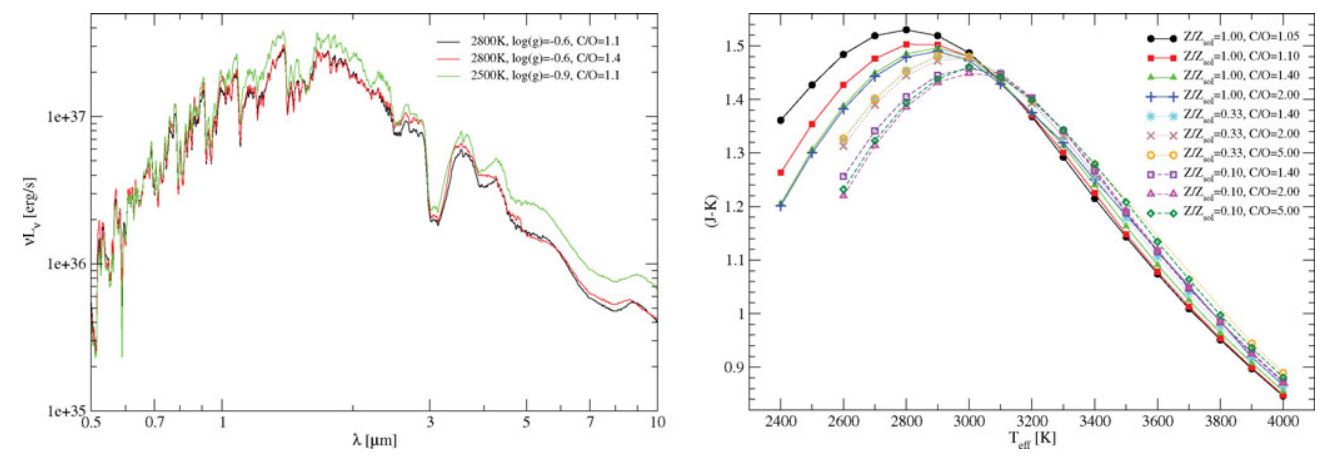

Figure 3. Left panel: Three spectra based on COMARCS models with $Z / Z_{\odot}=1.0$, $M / M_{\odot}=1.0$ and different values of $T_{\text {eff }}, \mathrm{C} / \mathrm{O}$ and $\log \left(g\left[\mathrm{~cm} / \mathrm{s}^{2}\right]\right)$. The resolution is $R=200$. Right panel: Predicted $J-K$ colours as a function of $T_{\text {eff }}$ calculated from COMARCS models with $\log \left(g\left[\mathrm{~cm} / \mathrm{s}^{2}\right]\right)=0, M / M_{\odot}=2$ and different values of $Z / Z_{\odot}\left(\right.$ denoted as $Z / Z_{\text {sol }}$ in the plot) and C/O. Adapted from Aringer et al. 2009

has been adapted (Girardi \& Marigo 2007b) so as to deal with the luminosity and $T_{\text {eff }}$ variations driven by thermal pulse cycles, and to consider additional variables such as the pulsation period of LPVs, the mass loss rate, the optical depth of circumstellar dust, etc. This allows us to simulate complete samples of AGB stars together with their main optical-to-infrared properties, for any history of star formation and chemical enrichment.

\section{TP-AGB stars in SSPs: trends with age and metallicity}

Broadband colours of Magellanic Cloud clusters represent the basic observable against which any population synthesis model should be compared for testing its predicting performance. TP-AGB stars are important contributors to their integrated bolometric and near-IR luminosities with a maximum located at an age of $\approx 1$ Gyr, accounting for about 40-80\% of the total cluster's luminosity (see Frogel et al. 1990; Maraston 2005). Interestingly, the position of the maximum reflects the peak in TP-AGB lifetimes at $M \approx 2.0-2.5 M_{\odot}$.

Due to the small number statistics of AGB stars in these clusters, stochastic fluctuations are of large entity, so that MCs' clusters cannot be assimilated to standard SSP of given age and metallicity. The best meaningful way to perform a comparison with observations is to simulate a population of clusters with the aid of Monte-Carlo techniques (Bruzual \& Charlot 2003).

Figure 4 compares the observed integrated $V-K$ colors of a sample of LMC clusters of known ages and a few predictions from different authors. Going from younger to older ages the first appearance of more massive AGB stars at $\approx 10^{8}$ Gyr produces a significant increase of colours like $V-K$ (e.g. Maraston 1998), with a rising slope that depends much on the details of processes like mass loss and HBB (Girardi \& Bertelli 1998). A further contribution to attain redder colours (e.g. $V-K, J-K$ ) is provided by C-stars, with a maximum effect at ages $\sim 0.5-1.0 \times 10^{9}$ yr corresponding to turn-off masses of $\sim 2.0-2.5 M_{\odot}$.

Figure 5 illustrates the predicted fractional contribution provided by TP-AGB stars to the integrated luminosities in different bands as a function of age and metallicity. It is evident that the trends change significantly at varying $Z$, suggesting that a mere extrapolation to other systems of the data suitable to metallicities typical of the MCs (i.e. $Z=0.004$ and $Z=0.008$ ) may lead to misleading results. 


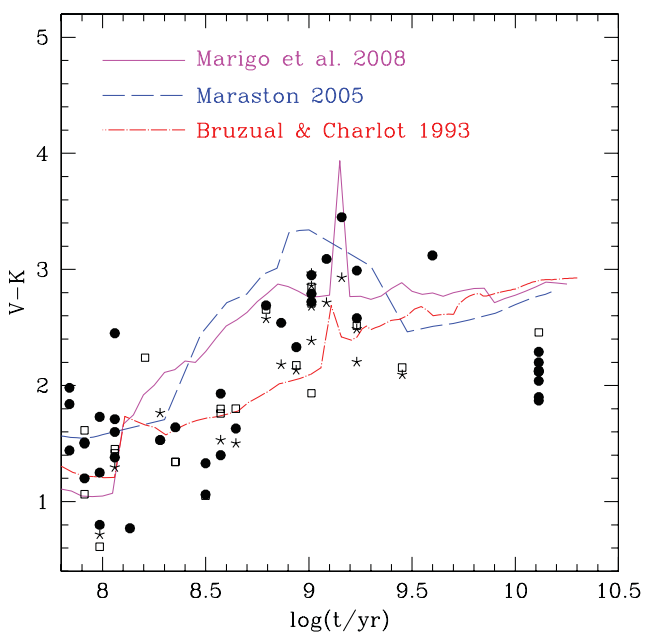

Figure 4. Broad-band $V-K$ colours of LMC clusters as a function of cluster age. Observed photometric data is taken from various compilations: Persson et al. (1983, filled circles); Kyeong et al. (2003, empty squares); and the $V$-band photometry by Goudfrooij et al. (2006) adopting an aperture radius of 50 arcsec, combined to the $J H K_{\mathrm{s}}$ photometry from Pessev et al. (2006; starred symbols). Clusters are assigned ages by adopting the $S$-parameter-age calibration by Girardi et al. (1995). Predicted SSP colours from various authors are superimposed for comparison: Marigo et al. (2008; $Z=0.008)$, Maraston $\left(2005 ; Z=Z_{\odot} / 2\right)$, Bruzual \& Charlot $(2003 ; Z=0.008)$.
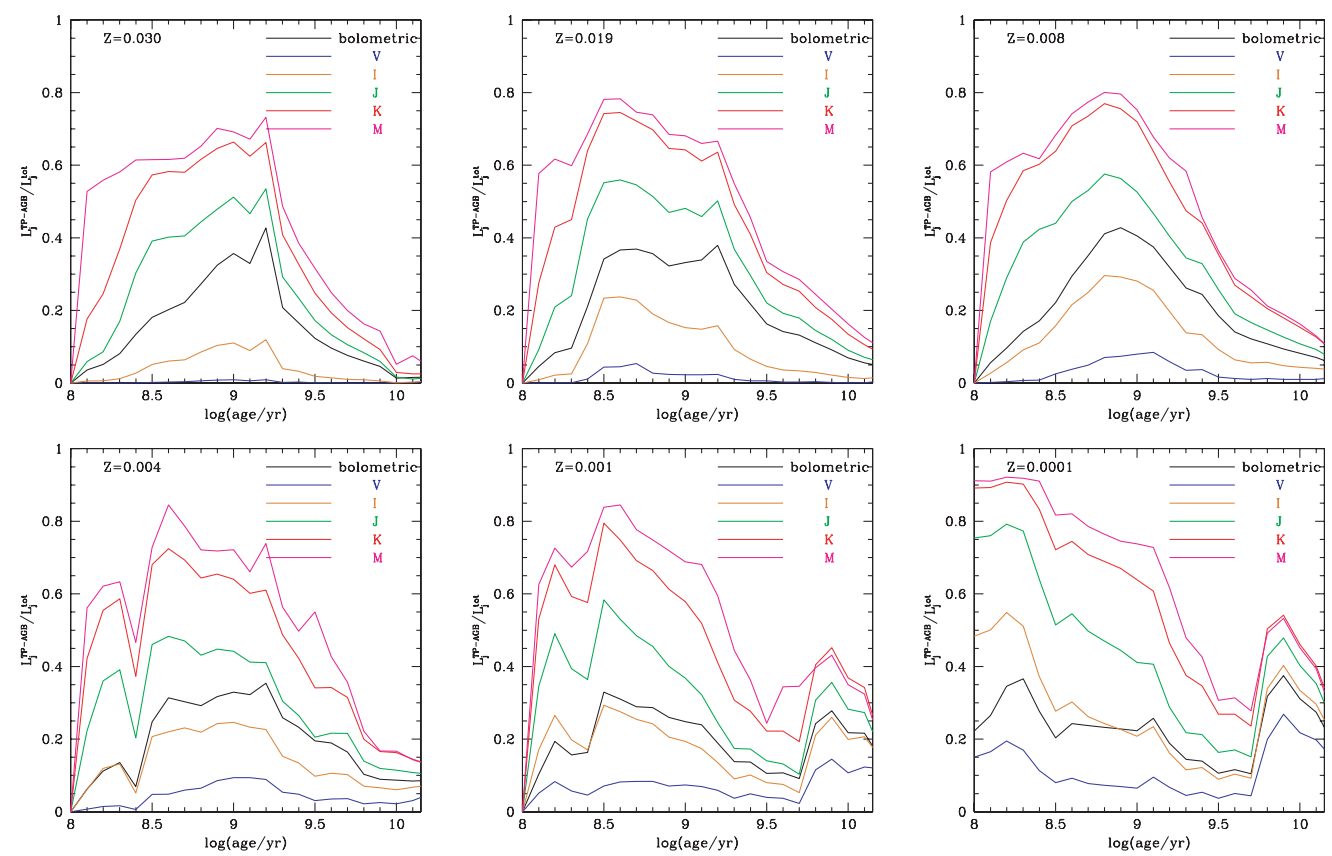

Figure 5. Fractional contribution from TP-AGB stars to the integrated luminosities in selected bands of simple stellar populations as a function of age and metallicity.

\section{Comparison with basic observables in star clusters and nearby galaxies}

We are presently dealing with simulations of the fields in the LMC and SMC, using published SFHs and AMRs (e.g. Harris \& Zaritsky 2004; Javiel et al. 2005; Pagel \& Tautvaisiene 1998). The models do a reasonable job in reproducing the number counts, magnitudes and colours of AGB stars in the Magellanic Clouds, which is no surprise since the evolutionary models have been originally calibrated using MC data. Looking at the quantitative details, however, we are accumulating several hints on the aspects of the models which need to be improved. 

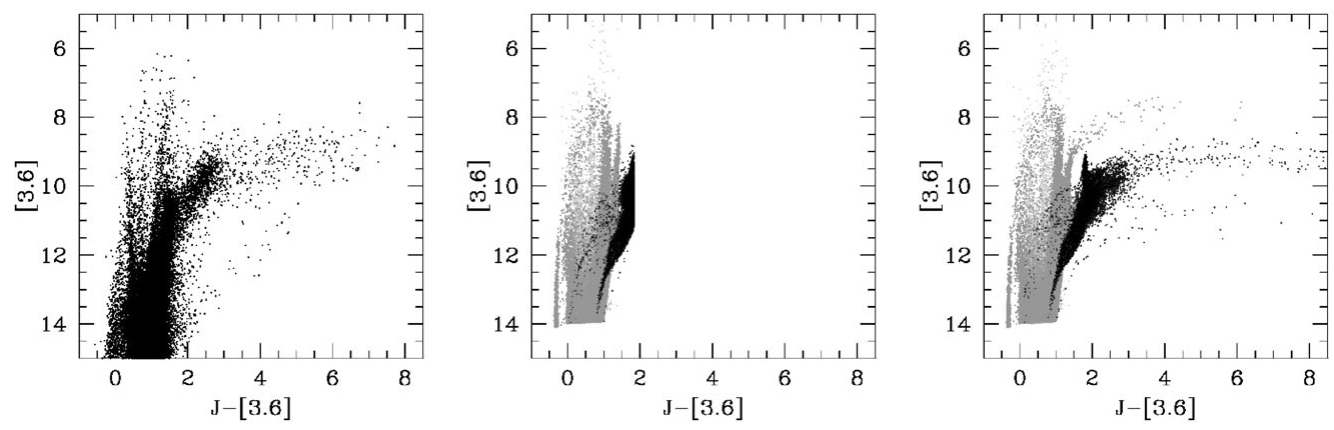

Figure 6. Comparison between Spitzer data for the LMC (Blum et al. 2006; left panel), and the simulated photometry without (middle panel) and with (right panel) the effect of AGB circumstellar dust. Predicted carbon stars are marked with black points.
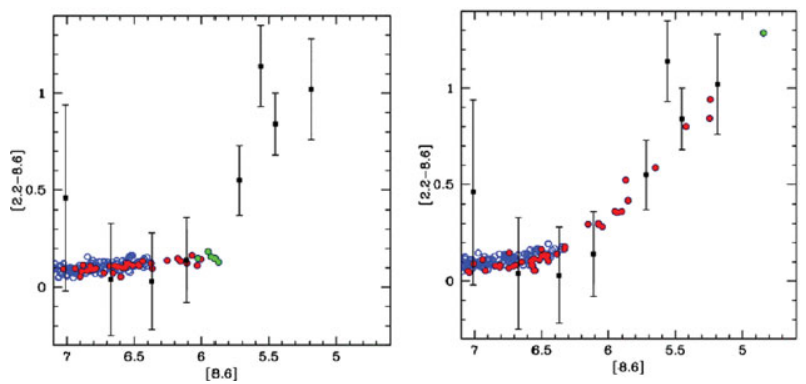

Figure 7. Comparison between observed (van Loon et al. 2006) and predicted CMDs for 47 Tuc in the near-mid IR. Simulations have been carried out without (left panel) and with (right panel) the effect of AGB circumstellar dust. TP-AGB stars are plotted with filled circles, and those with $\dot{M}>510^{-7} M_{\odot}$ $\mathrm{yr}^{-1}$ are marked in green.

Figs. 6 presents the typical results regarding the photometry of the LMC sample. While our models describe well the sequences of mass-losing stars in mid-infrared CMDs (Fig. 6), they fail to describe the detailed colour distributions. This likely means that we have to adjust the mass loss prescriptions, which, in turn, would imply the recalculation and recalibration of the TP-AGB tracks. We are implementing such iterative process, which is however not simple and quite demanding in terms of CPU time. No doubt the final target is well worth of the effort.

The old globular cluster 47 Tuc is of particular interest to us, thanks to the detailed information available for its AGB stars, and to its metallicity which is comparable to those found in young and intermediate-age populations of the MCs. Our simulations performed with the TRILEGAL code reproduce the behavior of the near and mid-IR colors that has been recently measured by van Loon et al. (2006) and Ita et al. (2007; AKARI data). As displayed in Figs. 7 the only stars that present a prominent mid-IR excess are those at the very tip of the AGB (see also van Loon et al. 2006; Lebzelter et al. 2006). This reinforces the idea that the bulk of dust formation occurs at the end of the AGB, with the role of RGB stars being quite marginal, if not negligible (see also Boyer et al. 2008, for $\omega$ Cen).

We are presently extending this kind of comparisons to the regime of low metallicities. Gullieuszik et al. (2008) and Held et al. (in prep.) have presented the first results for the LeoII and LeoI dSphs; they are summarised in Gullieuszik et al. (this volume). We have data for a few other dSphs observed with HAWK-I at VLT. Moreover, ongoing surveys with AO at Keck and the new HST/WFC3 camera (PIs: J. Melbourne and J. Dalcanton, respectively) will enormously increase the database of AGB stars in resolved dwarf galaxies. They will allow us to overcome the problem of small number statistics, at the price of dealing with somewhat more uncertain star formation histories. 
To conclude, we are entering a new era with both (1) much more detailed TP-AGB models and (2) more reliable, quantitative, and multi-wavelength constraints from AGB stars in resolved galaxies. This will help us to settle the TP-AGB contribution to the integrated light of galaxies on much firmer grounds, in the course of the next decade.

\section{Acknowledgements}

We acknowledge the financial support from INAF/PRIN07(CRA 1.06.10.03), the University of Padova (60A02-2949/09), MIUR/PRIN07 (prot. 20075TP5K9), and contract ASI-INAF I/016/07/0.

\section{References}

Aringer, B., Girardi, L., Nowotny, W., Marigo, P., \& Lederer, M. T. 2009, AESA, 503, 913

Blum, R. D., Mould, J. R., Olsen, K. A., et al. 2006, AJ, 132, 2034

Boyer, M. L., McDonald, I., van Loon, J. T. et al. 2008, AJ, 135, 1395

Bressan, A., Granato, G. L., \& Silva, L. 1998, A\& A, 332, 135

Bruzual, A. G. \& Charlot, S., 1993, ApJ, 405, 538

Frogel, J. A., Mould, J., \& Blanco, V. M. 1990, ApJ, 352, 96

Girardi, L. \& Marigo, P. 2007a, A\&A, 462, 237

Girardi, L. \& Marigo, P. 2007b, in Why galaxies care about AGB stars: their importance as actors and probes, eds. Kerschbaum, C. Charbonnel, \& R. F. Wing, ASP-CS, 378, p. 20, astro-ph/0701533

Girardi, L., Groenewegen, M. A. T., Hatziminaoglou, E. et al. 2005, A\& A, 436, 895

Girardi, L., Bressan, A., Bertelli, G. et al. 2000, A\&SAS, 141, 371

Girardi, L. \& Bertelli, G. 1998, MNRAS, 300, 533

Girardi, L., Chiosi, C., Bertelli, G., et al. 1995, A\&A, 298, 87

Goudfrooij, P., Gilmore, D., Kissler-Patig, M., \& Maraston, C. 2006, MNRAS, 369, 697

Groenewegen, M. A. T. 2006, $A \mathscr{E} A$ A, 448, 181

Groenewegen, M. A. T. 2002, ArXiv e-prints, astro-ph/0208449

Groenewegen, M. A. T. \& de Jong, T. 1993, A\& A, 267, 410

Gullieuszik M., Held E. V., Rizzi L. et al., 2008, MNRAS, 388, 1185

Harris, J. \& Zaritsky, D. 2004, AJ, 127, 1531

Ita, Y., Tanabé, T., Matsunaga, N., et al. 2007, PASJ, 59, 437

Javiel, S. C., Santiago, B. X., \& Kerber, L. O. 2005, A\&A, 431, 73

Kyeong, J.-M., Tseng, M.-J., \& Byun, Y.-I. 2003, A\&A, 409, 479

Lebzelter, T., Posch, T., Hinkle, K., Wood, P. R., \& Bouwman, J. 2006, ApJ, 653, L145

Loidl, R., Lançon, A., \& Jørgensen, U. G. 2001, A\& A, 371, 1065

Maraston, C. 2005, MNRAS, 362, 799

Maraston, C. 1998, MNRAS, 300, 872

Marigo, P. 2002, $A \mathscr{E} A, 387,507$

Marigo, P. \& Aringer B. 2009, arXiv:0907.3248

Marigo, P. \& Aringer, B. 2009, ArXiv e-prints, arXiv:0907.3248

Marigo, P., Girardi, L., Bressan, A., et al. 2008, A\& A, 482, 883

Marigo, P. \& Girardi, L. 2007, A $\& A, 469,239$

Marigo, P., Girardi, L., \& Bressan, A. 1999, A\& $\mathcal{G}$, 344, 123

Mouhcine, M. \& Lançon, A. 2003, A\& $A, 402,425$

Mouhcine, M. \& Lançon, A. 2002, A\&A, 393149

Pagel, B. E. J. \& Tautvaišienè, G. 1998, MNRAS, 299, 535

Pessev, P. M., Goudfrooij, P., Puzia., T. H., \& Chandar, R. 2006, AJ, 132, 781

Persson, S. E., Aaronson, M., Cohen, J. G., Frogel, J. A., \& Matthews, K. 1983,ApJ, 266, 105

Renzini, A. \& Buzzoni, A. 1986, in Spectral Evolution of Galaxies, C. Chiosi and A. Renzini (eds.), Dordrecht, Reidel, p. 195

van Loon, J. T., McDonald, I., Oliveira, J. M., et al. 2006, A\&̈A, 450, 339 